\title{
Influence of preformed plasma on surface-guided lateral transport of energetic electrons in ultraintense short laser-foil interactions
}

\author{
X H Yuan ${ }^{1,2, \dagger}$, D C Carroll ${ }^{2}$, J Zheng ${ }^{1}$, J L Liu ${ }^{1}$, R J Gray ${ }^{2}$, C \\ M Brenner ${ }^{2,4}$, M Coury ${ }^{2}$, L M Chen ${ }^{5}$, Y Fang ${ }^{1}$, O Tresca ${ }^{2}$, B \\ Zielbauer $^{3}, \mathbf{T} \mathbf{K u ̈ h l}^{3}, \mathrm{Y}$ T Li ${ }^{5}$, D Neely ${ }^{4,2}, \mathrm{Z}$ M Sheng ${ }^{1}, \mathbf{P}$ \\ McKenna $^{2, \dagger}$ \\ ${ }^{1}$ Key Laboratory for Laser Plasmas (Ministry of Education) and Department of \\ Physics and Astronomy, Shanghai Jiao Tong University, Shanghai 200240, China \\ ${ }^{2}$ SUPA Department of Physics, University of Strathclyde, Glasgow G4 0NG, UK \\ ${ }^{3}$ PHELIX Group, Gesellschaft für Schwerionenforschung mbH, Planckstrasse 1, \\ D-64291,Darmstadt, Germany \\ ${ }^{4}$ Central Laser Facility, Rutherford Appleton Laboratory, Oxfordshire OX11 0QX, \\ UK \\ ${ }^{5}$ Beijing National Laboratory of Condensed Matter Physics, Institute of Physics, \\ Chinese Academy of Sciences, Beijing 100190, China
}

\begin{abstract}
The lateral transport patterns of energetic electrons in thin foil targets irradiated by relativistically intense, picosecond laser pulses with different peak-topedestal intensity contrast ratios are reported. For 'low contrast' pulses, a large current of energetic electrons is found to be transported along the target front surface, due to the formation of strong quasi-static electric and magnetic fields. This is distinctly different from the case with 'high contrast' pulses, where energetic electrons are spatially confined. Although this lateral transport reduces laser energy coupling efficiency into ions and radiation production in the region of the laser focus, it can play an important role in directing energy transport in advanced fast ignition schemes involving hollow cone targets and also in heating the target (to generate states of warm dense matter) in regions far from the drive laser focus.
\end{abstract}

PACS numbers: 52.38.Fz, 52.38.Ph

† Email Address:xiaohui.yuan@sjtu.edu.cn

† Email Address:paul.mckenna@strath.ac.uk 


\section{Introduction}

The lateral transport (or spreading) of large currents of energetic ('fast') electrons in solids, generated by intense, short laser pulses, has important implications for many applications of laser-driven high energy density physics $[1,2,3,4,5,6]$. In the Fast Ignition approach to inertial confinement fusion (ICF), lateral spreading of the laseraccelerated fast electron population is detrimental to the efficient transfer of energy to the compressed fuel [7]. It could however play a beneficial role in directing energy transport along the surfaces of the hollow cone structures in advanced fusion targets [8]. Although the spreading of a fast electron beam in its transport through a thin foil generally reduces the magnitude of the rear-surface sheath acceleration field responsible for ion acceleration via the Target Normal Sheath Acceleration (TNSA) mechanism, lateral refluxing of electrons along the rear surface has been demonstrated to extend the field lifetime, and to 'shape' the ion beam by controlling the field evolution $[9,10]$. Laterally transported electrons also drive target heating, and thus the production of warm dense matter (WDM) states, in regions far from the laser focal spot [11].

Two main mechanisms have been proposed to explain lateral electron transport: (1) self-generated quasi-static electromagnetic fields on the target surface, which confine the electrons to a potential well, giving rise to a surface current $[1,4,5]$; and (2) fast electron recirculation or refluxing within the target, due to reflection by the sheath fields formed on both sides of the target, resulting in charge cloud expansion in the target bulk $[6,12]$. Despite the recent progress made, further work is required to determine the relative role that both mechanisms play, and their sensitivity to laser drive and target parameters.

In this Letter, we demonstrate that the intensity contrast ratio of the drive laser pulse is a key factor influencing the lateral transport of fast electrons. We find that the surface current dominates under low contrast conditions and that pile-up of the fast electrons on the target edges leads to efficient energy deposition on time-scales larger than the pulse duration.

\section{Experiment}

The experiment was performed using the PHELIX laser at the GSI laboratory in Germany. S-polarised laser pulses, with 0.5 ps duration (FWHM) and $1.054 \mu \mathrm{m}$ central wavelength, were focused using a $90^{\circ}$ off-axis $\mathrm{f} / 10$ parabolic mirror, onto foil targets, at an incident angle of $45^{\circ}$. The focal spot has an elliptical profile of $30 \mu \mathrm{m} \times 13 \mu \mathrm{m}$ (FWHM) in major and minor axes, respectively. The laser pulse energy on target was varied from $7 \mathrm{~J}$ to $90 \mathrm{~J}$, whilst maintaining the pulse duration and focal spot size, giving calculated peak intensities from $1 \times 10^{18}$ to $2 \times 10^{19} \mathrm{~W} / \mathrm{cm}^{2}$. The laser pulse intensity contrast, defined as the ratio of the intensity of the peak to that of the background pedestal, was controlled by detuning a Pockels cell in the laser chain. Two settings were chosen, to give measured pulse contrasts of $\sim 10^{7}$ and $\sim 10^{5}$, at 1 ns prior to the peak 
of the pulse, and these are referred to hereafter as the 'high contrast' and 'low contrast' cases.

Planar foil targets, in the shape of disk of $4 \mathrm{~mm}$ diameter and $20 \mu \mathrm{m}$ thickness, were used. The material was a copper (55\%) and nickel (45\%) alloy, glued onto aluminum mounting stalks. Figure 1(a) shows a schematic of the target assembly. The stalk overlaps a portion of the front surface of the target (to enable it to be fixed onto the stalk), but this region is far $(1 \mathrm{~mm})$ from the laser focus. Reference targets of $20 \mu \mathrm{m}-$ thick $\mathrm{Cu}$ foils $(2 \mathrm{~mm} \times 2 \mathrm{~mm})$ were also irradiated.

An imaging X-ray spectrometer was used to measure the K-shell transitions in both $\mathrm{Cu}$ and $\mathrm{Ni}$, by employing a high efficiency HOPG (Highly Oriented Pyrolitic Graphite) crystal and a double-entrance-slit. The double slits, both $30 \mu \mathrm{m}$ in width, are parallel to each other and perpendicular to the crystal surface, thus providing 1-D spatial measurement via pinhole imaging and 1-D spectral dispersion via Bragg diffraction. The design and characterization have been reported in detail elsewhere [13]. The spectrometer was positioned at $45^{\circ}$ to the target normal at the rear side and in the plane of the laser irradiation. The slits were orientated horizontally to enable spatial measurements in the vertical plane. At the front side of the target, a spherically-bent Bragg crystal was used to image the 2-D monochromic $\mathrm{Cu} \mathrm{K}_{\alpha}$ emission. The viewing angle was $20^{\circ}$ to the target normal axis. A pinhole camera was also used to monitor the continuum X-ray emission from the front side, close to the normal axis. Imaging Plate (IP) films (Fujifilm SR type) with dimensions of $5 \mathrm{~cm} \times 5 \mathrm{~cm}$ were used as detectors in all three diagnostics. Thin $(11 \mu \mathrm{m})$ aluminium foils were placed in front of the detectors to enable energy filtering and prevent direct exposure to the optical light.

Passive stacks of $200 \mathrm{~mm} \times 25 \mathrm{~mm}$ strips of dosimetry film (RCF: Gafchromic@film, HD-810) were bent to an open loop of inner diameter $11 \mathrm{~cm}$, centred on the target, to measure the spatial-intensity distribution of the lower half (just below the plane of the laser and the target normal axes) of the beam of accelerated protons. The proton energies corresponding to each piece of film were determined by calculating the proton energy loss through the stack. The arrangement of RCF (with part of a typical measurement showing the spatial-intensity distribution of the proton beam) is shown in Fig. 1 (a).

\section{Experimental results}

As shown in Fig. 1, the X-ray emission patterns measured for the two intensity contrast cases are significantly different, indicating different scenarios of fast electron transport. In the high contrast case (Fig. 1(b)), a single bright emission spot was observed at the target front side (Fig. 1(b)), with an approximately Gaussian intensity distribution of diameter $<200 \mu \mathrm{m}$ (FWHM). Note that only a small portion of image is shown, corresponding to a $650 \mu \mathrm{m} \times 650 \mu \mathrm{m}$ region of the target. In the low contrast case, shown in Fig. 1(c), the emission is not confined to a single spot, but is instead observed over a large (almost the entire) target area. Emission from the edges of the target and 


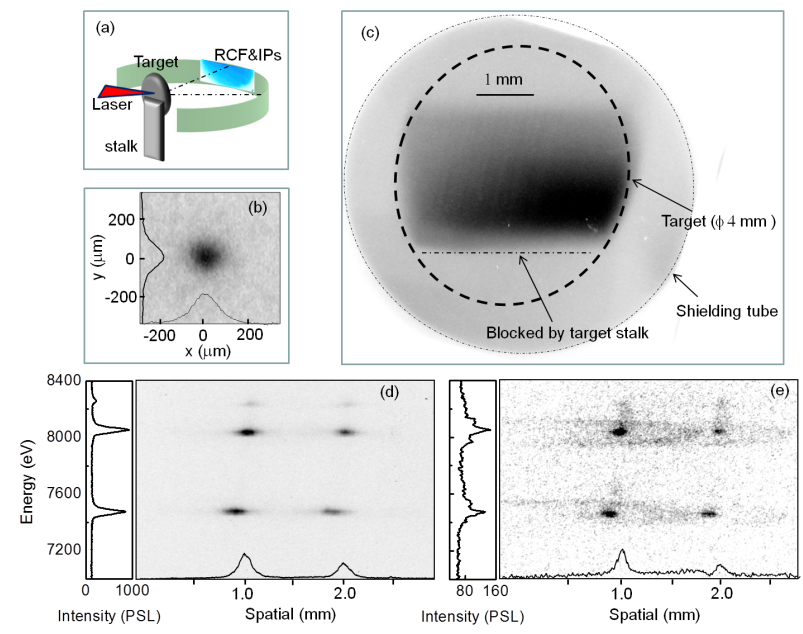

Figure 1. (color online). (a) Schematic illustration of the target assembly, showing laser irradiation direction and the positions of the RCF stacks. The main X-ray diagnostics are not shown (the positions of these are described in the main text). (b) Example measurement of the monochromatic $\mathrm{Cu} \mathrm{K}_{\alpha}$ emission in $2 \mathrm{D}$ with a high contrast $\left(\sim 10^{7}\right.$ at $\left.1 \mathrm{~ns}\right)$ laser pulse; (c) same as (b), but for low contrast $\left(\sim 10^{5}\right.$ at $1 \mathrm{~ns})$ laser pulse. Note that the laser is incident obliquely on the target, from the left side of these images. (d) Representative 1-D spatially-resolved spectrum from the rear side of the target with a high contrast laser pulse; (e) Same as (d), but for low contrast laser pulse irradiation. The laser energy is $\sim 60 \mathrm{~J}$ for both cases.

a shadow of the mounting stalk are well resolved. The total photon flux is twice that of the high contrast case when integrated across the whole target. Furthermore, the image is asymmetric, which is likely due to the oblique laser irradiation, as illustrated schematically in Fig. 1(a). The measurements of continuum X-ray emission, made with the pinhole camera, are consistent with these observations.

Figures 1(d) - (e) are representative measurements of 1-D spatially-resolved spectra of X-rays emitted from the target rear side, for the two contrast cases. The dual-slits produce two sets of profiles, spectrally dispersed in the vertical axis. For the high contrast case, well-defined emission lines on a faint background are measured, whereas the intensities of lines at similar laser pulse energies are much lower in the low contrast case. Furthermore, 'satellite' emission lines appear in the latter case, which are spatially extended, forming an asymmetric 'loop' around the brighter lines (the signal is stronger on the right side). This asymmetry is entirely consistent with the 2-D monochromatic X-ray images shown in Fig. 1(c), especially in terms of orientation.

The spectral line-outs are shown on the left panels of Figs. 1(d) and (e). The two main emission lines correspond to $\mathrm{Cu} \mathrm{K}_{\alpha}$ and $\mathrm{Ni} \mathrm{K}_{\alpha}$ transitions from the $\mathrm{Cu}-\mathrm{Ni}$ alloy target - the identification of these lines was confirmed by comparison to measurements made with $\mathrm{Cu}$ targets. The intensity of each $\mathrm{K}_{\alpha}$ line is almost one order of magnitude smaller for the low contrast case and the integrated flux of $K_{\alpha}$ photons, consisting of central hot spot and the 'satellite' lines, is about a factor of two smaller. The $\mathrm{Ni} \mathrm{K}_{\beta}$ is 
too weak to be measured for the low contrast laser shots. The spatial line-outs for the $\mathrm{Cu}$ $\mathrm{K}_{\alpha}$ line are shown on the bottom of Figs. 1(d) and (e). The 'satellite' emission region extends for several millimeters and corresponds to X-ray emission from an extended region of the target surface. Simulations were performed using the ray tracing code $\mathrm{XOP} / \mathrm{SHADOW}[14]$ to confirm this (simulation parameters are detailed in reference [13]). To simplify the modeling, the spatial profile of the X-ray source was designed as two points, orientated in the spectral dispersion plane. The photon energy is $8048 \mathrm{eV}$ (corresponding to $\mathrm{Cu} \mathrm{K}_{\alpha}$ ) and the spectral dispersion was modeled as a function of the separation of two sources (the two peaks only becoming distinguishable for separations greater than $\sim 160 \mu \mathrm{m}$ ). These simulations indicate that the separation of the sources, giving rise to the line-like features in the 'satellite' emission in Fig. 1(e), is the same as the target width and thus these features originate from X-ray emission from the target edges.

Considered together, the front and rear surface X-ray measurements indicate that under low contrast laser irradiation a significant proportion of the fast electrons generated at the front side of the target are guided along the front surface, continuously producing X-rays, and upon reaching the edges, due to the discontinuity in the available return current, they pile-up, enhancing X-ray production, before being reflected (on the timescale of the inverse of the plasma frequency) - the edge emission appearing as linelike features in the background 'satellite' emission in Fig. 1(e). The effect can also lead to the build-up of strong electric fields, resulting in ion acceleration at the target edges $[6]$.

Although in principle, electrons heated by the laser pulse pedestal (amplified spontaneous emission) may contribute to the surface current, we note that even for the case of the low contrast pulse, the pedestal intensity is only of the order of $\sim 10^{14} \mathrm{~W} / \mathrm{cm}^{2}$ which will produce an electron population with a temperature of $<6 \mathrm{keV}$, insufficient to account for the large number of $K_{\alpha}$ photons measured [12]. This, together with $\sim 90 \%$ reduction in $\mathrm{K}_{\alpha}$ flux emitted from the central hot spot at the target rear in the low contrast case, suggests that the larger preplasma expansion induced by the higherintensity pedestal acts to guide the main-peak generated fast electrons along the target front surface towards the edges [11].

Working in the weakly relativistic laser pulse peak intensity regime, $3 \times 10^{18}$ $\mathrm{W} / \mathrm{cm}^{2}$, and based on measurements of escaped fast electrons from foil targets, Habara et al [5] also infer significant surface transport of fast electrons in the present of preplasma. To investigate the sensitivity of this lateral electron transport to the drive laser pulse, we measured the X-ray emission for peak intensities between $1 \times 10^{18}$ and $2 \times 10^{19} \mathrm{~W} / \mathrm{cm}^{2}$. The integrated central hot spot signal of both the $\mathrm{Cu} \mathrm{K}_{\alpha}$ and $\mathrm{Ni} \mathrm{K}_{\alpha}$ (as measured using the HOPG spectrometer) as a function of laser intensity are presented in Fig 2.(a). The low contrast case produces consistently low central hot spot emission, and therefore significant lateral surface spreading, at all intensities sampled. Measurements of the maximum energy of TNSA-protons for the same laser shots, shown in Fig. 2(b), are fully consistent with a lower electron density in the central hot spot at the target rear 

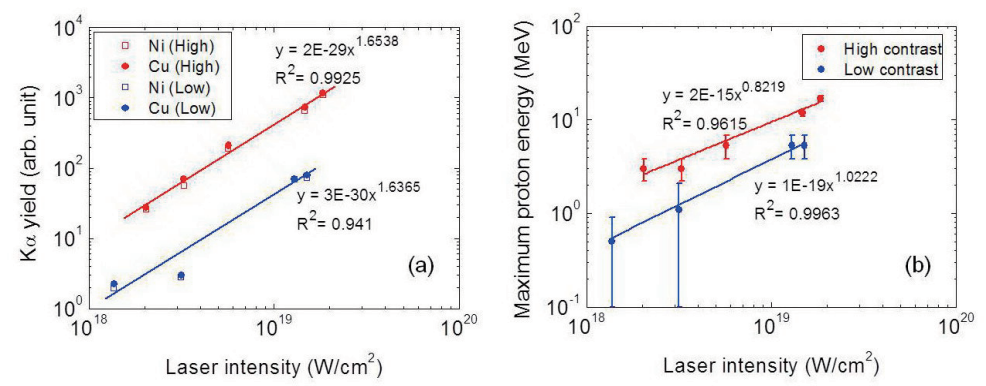

Figure 2. (color online). (a) Measured $\mathrm{K}_{\alpha}$ yield as a function of laser pulse intensity, for both $\mathrm{Ni}$ and $\mathrm{Cu}$, and for both the low and high contrast irradiation. (b) Maximum proton energy as a function of laser intensity for both contrast conditions.

due to significant lateral electron transport at the target front surface for the case of low contrast.

\section{Fast electron transport simulations}

To investigate the sensitivity of fast electron lateral transport in self-generated frontsurface-fields to the extent of the preformed plasma, 2D particle-in-cell (PIC) simulations with the KLAP-2D code [15] were performed. S-polarized laser pulses are incident at $45^{\circ}$ onto plasmas with two different scale lengths $\left(0 \lambda_{0}\right.$ and $2.07 \lambda_{0}$, where $\lambda_{0}$ is the laser wavelength in vacuum). In the first case, a step-like density profile is used with a constant density of $50 \mathrm{n}_{\mathrm{c}}$ (where $\mathrm{n}_{\mathrm{c}}$ is the critical density of the plasma) and length equal to $20 \lambda_{0}$. In the second case, the density increases exponentially from $0.4 \mathrm{n}_{\mathrm{c}}$ to $50 \mathrm{n}_{\mathrm{c}}$ in $10 \lambda_{0}$, and thereafter remains constant for a length of $20 \lambda_{0}$. The background ion species is protons. The lateral dimension of the targets is $80 \lambda_{0}$, and the overall size of the simulation box is $220 \lambda_{0} \times 200 \lambda_{0}$ in both cases. The boundaries are perfectly matched layer (PML) absorbing. The simulations were performed with 36 electrons per cell, and with cell size equal to $\lambda_{0} / 40$. The laser pulse ramps up with a sinusoidal profile within $10 \tau_{0}$, where $\tau_{0}$ is the laser period, maintains the intensity for $80 \tau_{0}$, and then decreases sinusoidally to zero in $10 \tau_{0}$. The normalized vector potential $\left(\mathrm{a}_{0}\right)$ is 3.0 , corresponding to an intensity of $1.24 \times 10^{19} \mathrm{~W} / \mathrm{cm}^{2}$ for $\lambda_{0}=1 \mu \mathrm{m}$. The laser focal spot is $10 \lambda_{0}$ in diameter (FWHM) with a Gaussian profile. The laser is incident from the left edge into the simulation box, along the $\mathrm{z}$-axis, and centered at $\left(\mathrm{x}=100 \lambda_{0}, \mathrm{z}=120 \lambda_{0}\right)$. The polarization direction is along the $\mathrm{X}$-axis. The leading edge of the pulse reaches the target front surface at time $\mathrm{t}=20 \tau_{0}$.

Figure 3 shows the phase space distributions of the fast electrons at three example time steps. At early time $t=30 \tau_{0}$ when the leading part of the laser pulse is reflected, high energy electrons are mainly constrained to the region of the laser focus in both cases, i.e. with and without preplasma. The electrons move predominately along the laser axis, and concentrate on the rear surface within a region with lateral dimension less than $40 \lambda_{0}$ for the case of no preplasma, at $t=110 \tau_{0}$. Only a small number of fast 

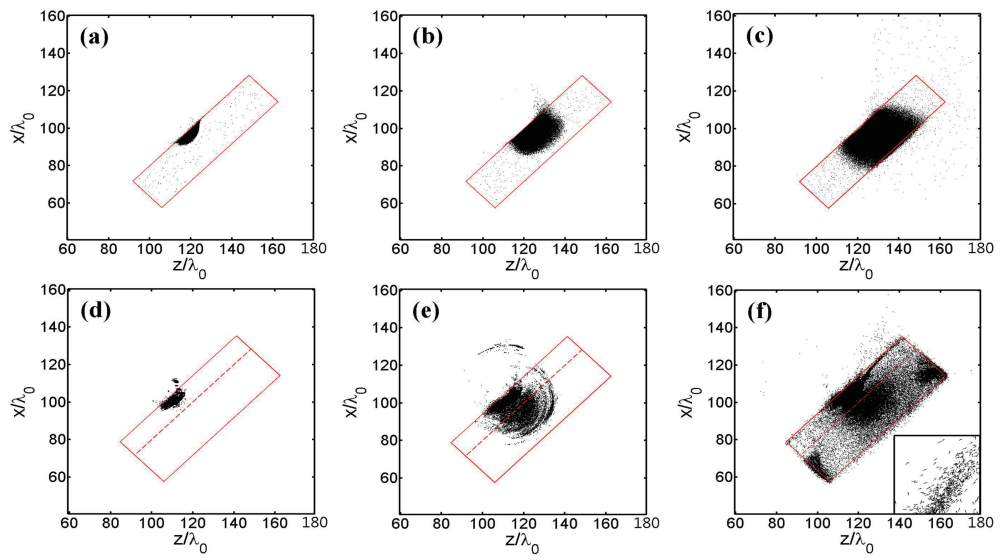

Figure 3. 2D PIC simulation results on phase space distributions of fast electrons at three time steps $\mathrm{t}=30 \tau_{0}(\mathrm{a}, \mathrm{d}), 60 \tau_{0}(\mathrm{~b}, \mathrm{e})$ and $110 \tau_{0}(\mathrm{c}, \mathrm{f})$, where $(\mathrm{a}-\mathrm{c})$ are for the case without preplasma and electrons are shown with $\gamma>1.1$, (d-f) are for the case with preplasma and electrons with $\gamma>8$. The different energies are selected because the temperature of the fast electron population is higher in the case of the preformed plasma, due to enhanced absorption. The box on each figure shows the initial target position and the dash line the boundary between the preplasma and constant density profiles. The inset in (f) plots the electron momentum vectors, which point mainly along the target surface.

electrons can reach the edges of the target at this time. However, the scenario changes when the laser interacts with preplasma. More than $30 \%$ of the electrons are confined in the preplasma layer at $\mathrm{t}=110 \tau_{0}$, flowing along the front surface, and a similar portion is collected on the target edge.

We also note that in addition to the surface current, fast electrons in the target bulk can also be transported to the target edge. The relative contributions of these two components to energy deposition at the target edge was quantified. At $\mathrm{t}=110 \tau_{0}$, the ratio of the surface-to-bulk transport components is 2 , indicating that the main lateral transport mechanism is the surface fields. We further note that more electrons appear on the top-right edge of the target than that of the bottom-left. This is consistent with the asymmetries measured with both the $\mathrm{Cu} \mathrm{K}$ imager and 1-D imaging spectroscopy.

A number of simulations were performed with the laser peak intensity varied and the preplasma profiles kept constant. The laterally transported fast electron current and deposition at the target edge were reproduced (in the preplasma case) independent of laser pulse intensity in the range explored in the experiment.

Figs. 4 shows the laser quasi-static electric and magnetic fields, at an example simulation time $t=110 \tau_{0}$, for the two cases investigated. In the absence of a preformed plasma, a significant fraction of the laser light is reflected. By contrast, in the case of a preformed plasma the laser beam filaments into multiple beamlets (as reported in a previous experiment [16]), driving deformation of the plasma critical density surface region and resulting in strong surface fields [17] and surface-directed electrons[1, 4]. 

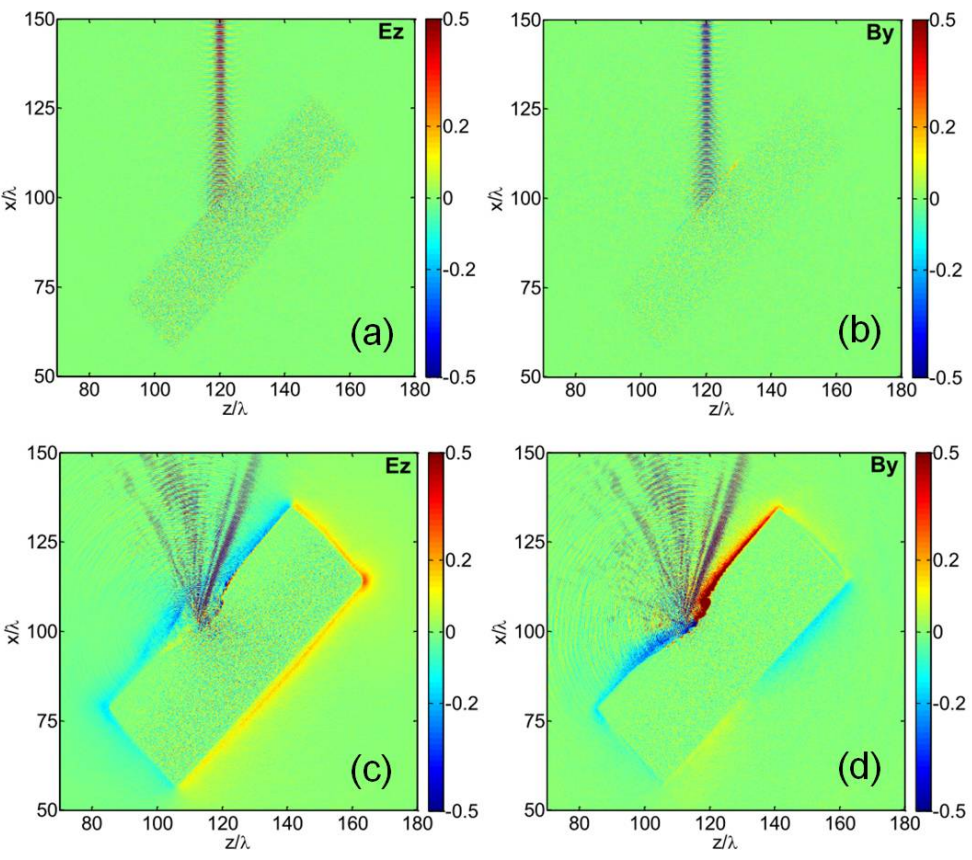

Figure 4. (color online). 2D simulation results showing: (a) and (c) the quasi-static electric field $\mathrm{E}_{\mathrm{z}}$; and $(\mathrm{b})$ and $(\mathrm{d})$ the magnetic field $\mathrm{B}_{\mathrm{y}}$ distributions, at $\mathrm{t}=110 \tau_{0}$. (a)(b) is the case without preplasma and (c)-(d) is the corresponding case with preplasma.

\section{Summary}

The demonstrated sensitivity of lateral fast electron transport to preformed plasma, over a wide peak laser intensity range, suggests that the effect is potentially switchable, which could enable better control of fast electron currents and energy deposition in many applications of high power laser-solid interactions, included advanced schemes for ICF.

We acknowledge expert support of the staff at the PHELIX laser facility at GSI, Darmstadt. This work was supported by EPSRC (grant nos. EP/J003832/1 and EP/L001357/1), National Basic Research Program of China (grant nos. 2013CBA01502 and 2013CBA01504), National Natural Science Foundation of China (grant nos. 11121504, 11205100 and 11220101002), and EU COST P-14 Action, LaserLAB Europe.

\section{References}

[1] Nakamura T et al 2004 Phys. Rev. Lett. 93265002.

[2] Mason R J et al 2005 Phys. Rev. E 72015401.

[3] Chen M et al 2006 Opt. Express 143093.

[4] Li Y T et al 2006 Phys. Rev. Lett. 96165003.

[5] Habara H et al 2006 Phys. Rev. Lett. 97095004.

[6] McKenna P et al 2007 Phys. Rev. Lett. 98145001.

[7] Tabak M et al 1994 Phys. Plasmas 11626.

[8] Kodama R et al 2001 Nature 412798. 
[9] Buffechoux S et al 2010 Phys. Rev. Lett. 105015005.

[10] Tresca O et al 2011 Plasma Phys. Control. Fusion 53105008.

[11] Baton S D et al 2007 High Energy Density Phys. 3358.

[12] Quinn M N et al 2011 Plasma Phys. Control. Fusion 53025007.

[13] Yuan X H et al 2011 Nucl. Instrum. Meth. A 653145.

[14] Rio M S d and Dejus R J 2004 SPIE Proc. 5536171.

[15] Chen M et al 2008 Chin. J. Comput. Phys. 2543.

[16] McKenna P et al 2008 Laser Part. Beams 26591.

[17] Yuan X H et al 2008 Phys. Plasmas 15013106. 